\title{
Addition theorems for the Appell polynomials and the associated classes of polynomial expansions
}

\author{
Á. Pintér and H. M. Srivastava
}

\begin{abstract}
Various interesting and potentially useful properties and relationships involving the Bernoulli, Euler and Genocchi polynomials have been investigated in the literature rather extensively. Recently, the present authors (Srivastava and Pinter in Appl Math Lett 17:375380 , 2004) obtained addition theorems and other relationships involving the generalized Bernoulli polynomials $B_{n}^{(\alpha)}(x)$ and the generalized Euler polynomials $E_{n}^{(\alpha)}(x)$ of order $\alpha$ and degree $n$ in $x$. The main purpose of this sequel to some of the aforecited investigations is to give several addition formulas for a general class of Appell sequences. The addition formulas, which are derived in this paper, involve not only the generalized Bernoulli polynomials $B_{n}^{(\alpha)}(x)$ and the generalized Euler polynomials $E_{n}^{(\alpha)}(x)$, but also the generalized Genocchi polynomials $G_{n}^{(\alpha)}(x)$, the Srivastava polynomials $\mathcal{S}_{n}^{N}(x)$, several general families of hypergeometric polynomials and such orthogonal polynomials as the Jacobi, Laguerre and Hermite polynomials. Some umbral-calculus generalizations of the addition formulas are also investigated.
\end{abstract}

Mathematics Subject Classification (2010). Primary 11B68; Secondary 11B83, 33C45.

Keywords. Addition theorems; Appell polynomials; Bernoulli, Euler and Genocchi polynomials; generating functions; Stirling numbers of the second kind; polynomial expansions; Srivastava polynomials; Jacobi, Laguerre and Hermite polynomials; hypergeometric polynomials.

\section{Introduction, definitions and preliminaries}

Throughout this paper, we use the following standard notations:

$$
\mathbb{N}:=\{1,2,3, \ldots\}, \quad \mathbb{N}_{0}:=\{0,1,2,3, \ldots\}=\mathbb{N} \cup\{0\}
$$

and

$$
\mathbb{Z}^{-}:=\{-1,-2,-3, \ldots\}=\mathbb{Z}_{0}^{-} \backslash\{0\} .
$$


Also, as usual, $\mathbb{Z}$ denotes the set of integers, $\mathbb{R}$ denotes the set of real numbers and $\mathbb{C}$ denotes the set of complex numbers.

The classical Bernoulli polynomials $B_{n}(x)$, the classical Euler polynomials $E_{n}(x)$ and the classical Genocchi polynomials $G_{n}(x)$, together with their familiar generalizations $B_{n}^{(\alpha)}(x), E_{n}^{(\alpha)}(x)$ and $G_{n}^{(\alpha)}(x)$ of (real or complex) order $\alpha$, are usually defined by means of the following generating functions (see, for details, [5, Vol. III, p. 253 et seq.], [10, Section 2.8] and [15, p. 61 et seq.]; see also [16, p. 81 et seq.] and [18] and the references cited therein):

$$
\begin{aligned}
& \left(\frac{t}{e^{t}-1}\right)^{\alpha} \cdot e^{x t}=\sum_{n=0}^{\infty} B_{n}^{(\alpha)}(x) \frac{t^{n}}{n !} \quad\left(|t|<2 \pi ; 1^{\alpha}:=1\right), \\
& \left(\frac{2}{e^{t}+1}\right)^{\alpha} \cdot e^{x t}=\sum_{n=0}^{\infty} E_{n}^{(\alpha)}(x) \frac{t^{n}}{n !} \quad\left(|t|<\pi ; 1^{\alpha}:=1\right)
\end{aligned}
$$

and

$$
\left(\frac{2 t}{e^{t}+1}\right)^{\alpha} \cdot e^{x t}=\sum_{n=0}^{\infty} G_{n}^{(\alpha)}(x) \frac{t^{n}}{n !} \quad\left(|t|<\pi ; 1^{\alpha}:=1\right)
$$

so that, obviously, the classical Bernoulli polynomials $B_{n}(x)$, the classical Euler polynomials $E_{n}(x)$ and the classical Genocchi polynomials $G_{n}(x)$ are given, respectively, by

$$
\begin{aligned}
B_{n}(x):=B_{n}^{(1)}(x), E_{n}(x):= & E_{n}^{(1)}(x) \text { and } G_{n}(x):=G_{n}^{(1)}(x) \\
& \left(n \in \mathbb{N}_{0}\right) .
\end{aligned}
$$

For the classical Bernoulli numbers $B_{n}$, the classical Euler numbers $E_{n}$ and the classical Genocchi numbers $G_{n}$ of order $n$, we have

$$
\begin{aligned}
B_{n}:=B_{n}(0)= & B_{n}^{(1)}(0), \quad E_{n}:=E_{n}(0)=E_{n}^{(1)}(0) \\
& \text { and } \quad G_{n}:=G_{n}(0)=G_{n}^{(1)}(0) \quad\left(n \in \mathbb{N}_{0}\right),
\end{aligned}
$$

respectively.

Various interesting and potentially useful properties and relationships involving the Bernoulli, Euler and Genocchi polynomials have been investigated in the literature rather extensively (see, in addition to the aforecited references, [1], [5, Vol. I, p. 35 et seq.] and [11]; see also [6,8,9] and the references cited therein). Recently, Chen et al. [4] presented several general classes of multiplication formulas and polynomial expansions in series of the Bernoulli and Euler polynomials for the Srivastava polynomials $\mathcal{S}_{n}^{N}(x)$ which are defined, in terms of a suitably bounded double sequence $\left\{A_{m, n}\right\}_{m, n \in \mathbb{N}_{0}}$ of essentially arbitrary (real or complex) parameters, by (cf. [14]; see also 
[7, p. 145, Equation (3.1) with $m=0])$

$$
\mathcal{S}_{n}^{N}(x):=\sum_{k=0}^{[n / N]} \frac{(-n)_{N k}}{k !} A_{n, k} x^{k} \quad\left(n \in \mathbb{N}_{0} ; N \in \mathbb{N}\right),
$$

where, as usual, $[\kappa]$ denotes the largest integer in $\kappa \in \mathbb{R}$ and $(\lambda)_{\nu}$ denotes the Pochhammer symbol or the shifted factorial, since

$$
(1)_{n}=n ! \quad\left(n \in \mathbb{N}_{0}\right),
$$

which is defined (for $\lambda, \nu \in \mathbb{C}$ ), in terms of the familiar Gamma function, by

$$
(\lambda)_{\nu}:=\frac{\Gamma(\lambda+\nu)}{\Gamma(\lambda)}= \begin{cases}1 & (\nu=0 ; \lambda \in \mathbb{C} \backslash\{0\}) \\ \lambda(\lambda+1) \cdots(\lambda+n-1) & (\nu=n \in \mathbb{N} ; \lambda \in \mathbb{C}),\end{cases}
$$

it being understood conventionally that $(0)_{0}:=1$. For example, Chen et al. [4] derived the following multiplication formulas and applied each of their results to hypergeometric polynomials as well as many classical orthogonal polynomials including (for example) the Jacobi, Laguerre and Hermite polynomials [4, p. 137, Equation (2.5); p. 140, Equation (2.24)]:

$$
\begin{aligned}
\mathcal{S}_{n}^{N}(\omega x)= & \sum_{k=0}^{[n / N]}(-1)^{N k}\left(\begin{array}{c}
n \\
N k
\end{array}\right) \frac{(N k) !}{k !} \\
& \cdot\left(\sum_{j=0}^{[(n-N k) / N]} \frac{(-n+N k)_{N j}}{(j+1) !} A_{n, j+k} \omega^{j+k}\right) B_{k}(x) \\
& \left(n \in \mathbb{N}_{0} ; N \in \mathbb{N}\right)
\end{aligned}
$$

and

$$
\begin{aligned}
\mathcal{S}_{n}^{N}(\omega x)= & \frac{1}{2} \sum_{k=0}^{[n / N]}(-1)^{N k}\left(\begin{array}{c}
n \\
N k
\end{array}\right) \frac{(N k) !}{k !} \\
& \cdot\left(A_{n, k}+\sum_{j=0}^{[(n-N k) / N]} \frac{(-n+N k)_{N j}}{j !} A_{n, j+k} \omega^{j}\right) \omega^{k} E_{k}(x) \\
& \left(n \in \mathbb{N}_{0} ; N \in \mathbb{N}\right) .
\end{aligned}
$$

More recently, the present authors [18] obtained addition theorems and other relationships involving the generalized Bernoulli polynomials $B_{n}^{(\alpha)}(x)$ and the generalized Euler polynomials $E_{n}^{(\alpha)}(x)$. The purpose of this sequel to some of the aforecited investigations is to give several addition formulas for a general class of Appell sequences. 
Definition 1 (see [3]; see also [12, p. 145, Exercise 2] and [17, p. 398, Problem 28].) A polynomial sequence $\left\{f_{n}(x)\right\}_{n \in \mathbb{N}_{0}}$ is said to be an Appell sequence if

$$
\frac{d}{d x}\left\{f_{0}(x)\right\}=0 \quad \text { and } \quad \frac{d}{d x}\left\{f_{n}(x)\right\}=n f_{n-1}(x) \quad(n \in \mathbb{N})
$$

or, equivalently, if

$$
f(t) \cdot e^{x t}=\sum_{n=0}^{\infty} f_{n}(x) \frac{t^{n}}{n !} \quad(f(0) \neq 0) .
$$

Some obvious examples for Appell sequences are the trivial monomials $\left\{x^{n}\right\}_{n \in \mathbb{N}_{0}}$ as well as the generalized Bernoulli polynomials $B_{n}^{(\alpha)}(x)$ and the generalized Euler polynomials $E_{n}^{(\alpha)}(x)$ which are defined by their exponential generating functions (1) and (2) by choosing

$$
f(t)=\left(\frac{t}{e^{t}-1}\right)^{\alpha} \quad \text { and } \quad f(t)=\left(\frac{2}{e^{t}+1}\right)^{\alpha},
$$

respectively, in the defining generating function (10) above. Other examples of Appell sequences are the monic Hermite polynomials $\left\{2^{-n} H_{n}(x)\right\}_{n \in \mathbb{N}_{0}}$ and the modified Laguerre polynomials $\left\{(-1)^{n} n ! L_{n}^{(\alpha-n)}(x)\right\}_{n \in \mathbb{N}_{0}}$ for which (see, for details [17, Section 1.11])

$$
f(t)=\exp \left(-\frac{t^{2}}{4}\right) \quad \text { and } \quad f(t)=(1-t)^{\alpha},
$$

respectively, in the defining generating function (10) above.

Remark 1. Other definitions and notations for Appell sequences can be found in the existing literature (see, for example [2]).

In our present investigation, we shall also need the Stirling numbers $S(n, k)$ of the second kind defined by means of the following expansion (see [15, p. 58 et seq.]):

$$
x^{n}=\sum_{k=0}^{n}\left(\begin{array}{l}
x \\
k
\end{array}\right) k ! S(n, k)
$$

or, equivalently, by the following generating functions:

$$
\left(e^{t}-1\right)^{k}=k ! \sum_{n=k}^{\infty} S(n, k) \frac{t^{n}}{n !}
$$

and

$$
(1-t)^{-1}(1-2 t)^{-1} \cdots(1-k t)^{-1}=\sum_{n=k}^{\infty} S(n, k) t^{n-k} \quad\left(|t|<k^{-1}\right)
$$


where $S(n, k)$ denotes the number of ways of partitioning a set of $n$ elements into $k$ non-empty subsets, so that

$$
S(n, 0)=\delta_{n, 0}, \quad S(n, 1)=S(n, n)=1 \quad \text { and } \quad S(n, n-1)=\left(\begin{array}{l}
n \\
2
\end{array}\right),
$$

$\delta_{n, k}$ being the Kronecker symbol.

\section{The first set of addition formulas}

With a view to use it in deriving our first set of addition formulas for the Appell polynomials $\left\{f_{n}(x)\right\}_{n \in \mathbb{N}_{0}}$ given by (9) and (10), we present the following lemma.

Lemma 1. Each of the following expansion formulas holds true:

$$
\begin{aligned}
x^{n} & =\sum_{k=0}^{n} \frac{n !}{k !(n-k+1) !} B_{k}(x), \\
x^{n} & =\frac{1}{2}\left[E_{n}(x)+\sum_{k=0}^{n}\left(\begin{array}{l}
n \\
k
\end{array}\right) E_{k}(x)\right]
\end{aligned}
$$

and

$$
x^{n}=\frac{1}{2(n+1)}\left[G_{n+1}(x)+\sum_{k=0}^{n}\left(\begin{array}{l}
n+1 \\
k+1
\end{array}\right) G_{k+1}(x)\right] .
$$

Proof. The expansion formulas (16) and (17) are well known (see, for example, [18, p. 378, Equations (27) and (29)]). In order to demonstrate the assertion (18) of Lemma 1, we easily find from the generating function (3) that

$$
G_{n}^{(\alpha+\beta)}(x+y)=\sum_{k=0}^{\infty}\left(\begin{array}{l}
n \\
k
\end{array}\right) G_{k}^{(\alpha)}(x) G_{n-k}^{(\beta)}(y)
$$

which, in the special case when $y=1$ and $\beta=0$, yields

$$
G_{n}^{(\alpha+\beta)}(x+1)=\sum_{k=0}^{\infty}\left(\begin{array}{l}
n \\
k
\end{array}\right) G_{k}^{(\alpha)}(x) .
$$

Moreover, it readily follows from the generating function (3) (with $\alpha=1$ ) that

$$
G_{n+1}(x+1)+G_{n+1}(x)=2(n+1) x^{n} \quad\left(n \in \mathbb{N}_{0}\right) .
$$

Thus, upon combining (20) and (21), we are led to the assertion (18) of Lemma 1.

Alternatively, since (see, for example, [9, p. 5707, Lemma 1])

$$
\begin{aligned}
G_{n}^{(\ell)}(x)= & \frac{n !}{(n-\ell) !} E_{n-\ell}^{(\ell)}(x)=\left(\begin{array}{l}
n \\
\ell
\end{array}\right) \ell ! E_{n-\ell}^{(\ell)}(x) \\
& \left(n, \ell \in \mathbb{N}_{0} ; 0 \leqq \ell \leqq n\right)
\end{aligned}
$$


or, equivalently,

$$
E_{n}^{(\ell)}(x)=\frac{n !}{(n+\ell) !} G_{n+\ell}^{(\ell)}(x)=\frac{1}{\ell !}\left(\begin{array}{c}
n+\ell \\
\ell
\end{array}\right)^{-1} G_{n+\ell}^{(\ell)}(x) \quad\left(n, \ell \in \mathbb{N}_{0}\right),
$$

so that, obviously,

$$
E_{n}(x)=\frac{1}{n+1} G_{n+1}(x) \quad \text { and } \quad G_{n}(x)=n E_{n-1}(x),
$$

the assertion (18) of Lemma 1 involving the Genocchi polynomials $G_{n}(x)$ can be deduced from the assertion (17) of Lemma 1 involving the Euler polynomials $E_{n}(x)$.

Remark 2. Making use of the expansion formula (18) or (alternatively) by means of the first relationship in (24), we get the following companion of the multiplication formulas (7) and (8) for the Srivastava polynomials $\mathcal{S}_{n}^{N}(x)$ defined by (6):

$$
\begin{aligned}
\mathcal{S}_{n}^{N}(\omega x)= & \frac{1}{2} \sum_{k=0}^{[n / N]}(-1)^{N k}\left(\begin{array}{c}
n \\
N k
\end{array}\right) \frac{(N k) !}{(k+1) !} \\
& \cdot\left(A_{n, k}+\sum_{j=0}^{[(n-N k) / N]} \frac{(-n+N k)_{N j}}{j !} A_{n, j+k} \omega^{j}\right) \omega^{k} G_{k+1}(x) \\
& \left(n \in \mathbb{N}_{0} ; N \in \mathbb{N}\right) .
\end{aligned}
$$

involving the Genocchi polynomials $G_{n}(x)$.

We choose to first state our addition formulas in terms of the one-parameter Appell sequence $\left\{f_{n}^{(\alpha)}(x)\right\} \quad(\alpha \in \mathbb{C})$ generated by

$$
(f(t))^{\alpha} \cdot e^{x t}=\sum_{n=0}^{\infty} f_{n}^{(\alpha)}(x) \frac{t^{n}}{n !} \quad\left(f(0) \neq 0 ; 1^{\alpha}:=1\right),
$$

so that, by comparing with the generating function (10), we have

$$
f_{n}^{(1)}(x)=f_{n}(x) \quad\left(n \in \mathbb{N}_{0}\right) .
$$

Obviously, the generating function (26) is much more in line with the generating functions (1) and (2) for the Bernoulli and Euler polynomials of (real or complex) order $\alpha$. In fact, the one-parameter Appell sequence $\left\{f_{n}^{(\alpha)}(x)\right\} \quad(\alpha \in$ $\mathbb{C})$ can easily and precisely be identified with the generalized Bernoulli polynomials $B_{n}^{(\alpha)}(x)$, the generalized Euler polynomials $E_{n}^{(\alpha)}(x)$ and the modified Laguerre polynomials $(-1)^{n} n ! L_{n}^{(\alpha)}(x)$ for which

$$
f(t)=\frac{t}{e^{t}-1}, \quad f(t)=\frac{2}{e^{t}+1} \quad \text { and } \quad f(t)=(1-t),
$$

respectively. 
Theorem 1. Let $\left\{f_{n}^{(\alpha)}(x)\right\}_{n \in \mathbb{N}_{0}}$ be a one-parameter sequence of Appell polynomials. generated by (26). Then each of the following addition formulas holds true:

$$
\begin{aligned}
f_{n}^{(\alpha)}(x+y)= & \sum_{k=0}^{n}\left[\sum_{j=k}^{n} \frac{1}{j+1}\left(\begin{array}{c}
n \\
j
\end{array}\right)\left(\begin{array}{c}
j+1 \\
k
\end{array}\right) f_{n-j}^{(\alpha)}(y)\right] B_{k}(x), \\
f_{n}^{(\alpha)}(x+y)= & \frac{1}{2} \sum_{k=0}^{n}\left[\left(\begin{array}{l}
n \\
k
\end{array}\right) f_{n-k}(y)+\sum_{j=k}^{n}\left(\begin{array}{l}
n \\
j
\end{array}\right)\left(\begin{array}{l}
j \\
k
\end{array}\right) f_{n-j}^{(\alpha)}(y)\right] E_{k}(x), \\
f_{n}^{(\alpha)}(x+y)= & \frac{1}{2} \sum_{k=0}^{n} \frac{1}{k+1}\left[\left(\begin{array}{l}
n \\
k
\end{array}\right) f_{n-k}^{(\alpha)}(y)+\sum_{j=k}^{n}\left(\begin{array}{l}
n \\
j
\end{array}\right)\left(\begin{array}{l}
j \\
k
\end{array}\right) f_{n-j}^{(\alpha)}(y)\right] \\
& \cdot G_{k+1}(x)
\end{aligned}
$$

and

$$
f_{n}^{(\alpha)}(x+y)=\sum_{k=0}^{n} k !\left[\sum_{j=k}^{n}\left(\begin{array}{l}
n \\
j
\end{array}\right) S(j, k) f_{n-j}^{(\alpha)}(y)\right]\left(\begin{array}{l}
x \\
k
\end{array}\right) .
$$

Proof. For the one-parameter Appell sequence $\left\{f_{n}^{(\alpha)}(x)\right\}_{n \in \mathbb{N}_{0}}$ generated by (26), it is easily observed that

$$
f_{n}^{(\alpha+\beta)}(x+y)=\sum_{k=0}^{n}\left(\begin{array}{l}
n \\
k
\end{array}\right) f_{k}^{(\alpha)}(x) f_{n-k}^{(\beta)}(y),
$$

so that, since

$$
f_{n}^{(0)}(x)=x^{n} \quad\left(n \in \mathbb{N}_{0}\right)
$$

we have

$$
f_{n}^{(\alpha)}(x+y)=\sum_{k=0}^{n}\left(\begin{array}{l}
n \\
k
\end{array}\right) f_{n-k}^{(\alpha)}(y) x^{k} .
$$

Now, upon replacing the factor $x^{k}$ in (31) by its expansion given by the assertion (16) of Lemma 1, if we interchange the order of the resulting double sum, we arrive at the assertion (27) of Theorem 1.

The assertions (28) and (29) of Theorem 1 can be proven similarly by using the expansion formulas (17) and (18) in (32). Of course, in view of the relationships stated in (24), the assertions (28) and (29) of Theorem 1 are essentially equivalent.

The last assertion (30) of Theorem 1 is a similar consequence of (32) and the definition (12) of Stirling numbers of the second kind.

Upon setting $\alpha=1$, Theorem 1 yields the following corollary. 
Corollary 1. Let $\left\{f_{n}(x)\right\}_{n \in \mathbb{N}_{0}}$ be an arbitrary sequence of Appell polynomials. Then each of the following addition formulas holds true:

$$
\begin{aligned}
f_{n}(x+y)= & \sum_{k=0}^{n}\left[\sum_{j=k}^{n} \frac{1}{j+1}\left(\begin{array}{c}
n \\
j
\end{array}\right)\left(\begin{array}{c}
j+1 \\
k
\end{array}\right) f_{n-j}(y)\right] B_{k}(x), \\
f_{n}(x+y)= & \frac{1}{2} \sum_{k=0}^{n}\left[\left(\begin{array}{l}
n \\
k
\end{array}\right) f_{n-k}(y)+\sum_{j=k}^{n}\left(\begin{array}{l}
n \\
j
\end{array}\right)\left(\begin{array}{l}
j \\
k
\end{array}\right) f_{n-j}(y)\right] E_{k}(x), \\
f_{n}(x+y)= & \frac{1}{2} \sum_{k=0}^{n} \frac{1}{k+1}\left[\left(\begin{array}{l}
n \\
k
\end{array}\right) f_{n-k}(y)+\sum_{j=k}^{n}\left(\begin{array}{l}
n \\
j
\end{array}\right)\left(\begin{array}{l}
j \\
k
\end{array}\right) f_{n-j}(y)\right] \\
& \cdot G_{k+1}(x)
\end{aligned}
$$

and

$$
f_{n}(x+y)=\sum_{k=0}^{n} k !\left[\sum_{j=k}^{n}\left(\begin{array}{l}
n \\
j
\end{array}\right) S(j, k) f_{n-j}(y)\right]\left(\begin{array}{l}
x \\
k
\end{array}\right) .
$$

If, in Theorem 1 , we set $\alpha=1$ and $x=0$, we are led immediately to the following corollary involving the Bernoulli, Euler and Genocchi numbers defined by (5).

Corollary 2. Let $\left\{f_{n}(x)\right\}_{n \in \mathbb{N}_{0}}$ be an arbitrary sequence of Appell polynomials. Then each of the following addition formulas holds true:

$$
\begin{aligned}
& f_{n}(x)=\sum_{k=0}^{n}\left[\sum_{j=k}^{n} \frac{1}{j+1}\left(\begin{array}{c}
n \\
j
\end{array}\right)\left(\begin{array}{c}
j+1 \\
k
\end{array}\right) f_{n-j}(x)\right] B_{k}, \\
& f_{n}(x)=\frac{1}{2} \sum_{k=0}^{n}\left[\left(\begin{array}{l}
n \\
k
\end{array}\right) f_{n-k}(x)+\sum_{j=k}^{n}\left(\begin{array}{l}
n \\
j
\end{array}\right)\left(\begin{array}{l}
j \\
k
\end{array}\right) f_{n-j}(x)\right] E_{k}
\end{aligned}
$$

and

$$
f_{n}(x)=\frac{1}{2} \sum_{k=0}^{n} \frac{1}{k+1}\left[\left(\begin{array}{l}
n \\
k
\end{array}\right) f_{n-k}(x)+\sum_{j=k}^{n}\left(\begin{array}{l}
n \\
j
\end{array}\right)\left(\begin{array}{l}
j \\
k
\end{array}\right) f_{n-j}(x)\right] G_{k+1} .
$$

\section{Addition formulas involving hypergeometric polynomials}

For two general families of hypergeometric polynomials, Chen et al. [4] derived the following expansion formulas (see, for details [4, p. 149, Equations 
(3.39) and (3.40)]):

$$
\begin{aligned}
& x^{n}=\frac{n !}{(N n) !}\left(\frac{\prod_{j=1}^{q}\left(\beta_{j}\right)_{n} \prod_{j=1}^{r}\left(\gamma_{j}\right)_{N n}}{\prod_{j=1}^{p}\left(\alpha_{j}\right)_{n} \prod_{j=1}^{s}\left(\delta_{j}\right)_{N n}}\right) \sum_{k=0}^{N n}(-1)^{k}\left(\begin{array}{c}
N n \\
k
\end{array}\right) \frac{\lambda+2 k}{(\lambda+k)_{N n+1}} \\
& \cdot{ }_{p+N(s+2)} F_{q+N r}\left[\begin{array}{c}
\Delta(N ;-k), \Delta(N ; \lambda+k),\left(\alpha_{p}\right), \Delta\left[N ;\left(\delta_{s}\right)\right] ; x N^{(s-r+2) N} \\
\left(\beta_{q}\right), \Delta\left[N ;\left(\gamma_{r}\right)\right] ;
\end{array}\right]
\end{aligned}
$$

and

$$
\begin{aligned}
x^{n}= & \frac{n !}{(N n) !}\left(\frac{\prod_{j=1}^{q}\left(\beta_{j}\right)_{n} \prod_{j=1}^{r}\left(\gamma_{j}\right)_{N n}}{\prod_{j=1}^{p}\left(\alpha_{j}\right)_{n} \prod_{j=1}^{s}\left(\delta_{j}\right)_{N n}}\right) \sum_{k=0}^{N n}(-1)^{k}\left(\begin{array}{c}
N n \\
k
\end{array}\right) \\
& \cdot{ }_{p+N(s+1)} F_{q+N r}\left[\begin{array}{c}
\Delta(N ;-k),\left(\alpha_{p}\right), \Delta\left[N ;\left(\delta_{s}\right)\right] ; \\
\left(\beta_{q}\right), \Delta\left[N ;\left(\gamma_{r}\right)\right] ;
\end{array}\right],
\end{aligned}
$$

where, for convenience, we use such contracted notations as (for example) $\left(\alpha_{p}\right)$ for the array of $p$ parameters

$$
\alpha_{1}, \ldots, \alpha_{p}
$$

$\Delta(N ; \lambda)$ for the array of $N$ parameters

$$
\frac{\lambda}{N}, \frac{\lambda+1}{N}, \ldots, \frac{\lambda+N-1}{N} \quad(N \in \mathbb{N}),
$$

$\Delta\left[N ;\left(\alpha_{p}\right)\right]$ for the array of $N p$ parameters

$$
\frac{\alpha_{j}}{N}, \frac{\alpha_{j}+1}{N}, \ldots, \frac{\alpha_{j}+N-1}{N} \quad(j=1, \ldots, p ; N \in \mathbb{N})
$$

and so on, an empty product being interpreted (as usual) to be 1 .

By applying the expansion formulas (40) and (41) in conjunction with (32), it is not difficult to prove Theorem 2 below.

Theorem 2. Let $\left\{f_{n}^{(\mu)}(x)\right\}_{n \in \mathbb{N}_{0}}$ be a one-parameter sequence of Appell polynomials generated by (26) with the parameter $\alpha$ replaced by $\mu$. Then each of the following addition formulas holds true for $N \in \mathbb{N}$ : 


$$
\begin{aligned}
& f_{n}^{(\mu)}(x+y)=\sum_{k=0}^{N n}(-1)^{k} \sum_{j=[k / N]}^{n} \frac{j !}{(N j) !}\left(\begin{array}{c}
n \\
j
\end{array}\right)\left(\begin{array}{c}
N j \\
k
\end{array}\right) \\
& \cdot \frac{\lambda+2 k}{(\lambda+k)_{N j+1}}\left(\frac{\prod_{l=1}^{q}\left(\beta_{l}\right)_{j} \prod_{l=1}^{r}\left(\gamma_{l}\right)_{N j}}{\prod_{l=1}^{p}\left(\alpha_{l}\right)_{j} \prod_{l=1}^{s}\left(\delta_{l}\right)_{N j}}\right) f_{n-j}^{(\mu)}(y) \cdot{ }_{p+N(s+2)} F_{q+N r} \\
& \quad \times\left[\begin{array}{c}
\Delta(N ;-k), \Delta(N ; \lambda+k),\left(\alpha_{p}\right), \Delta\left[N ;\left(\delta_{s}\right)\right] ; \\
\left.x N^{(s-r+2) N}\right]
\end{array}\right]
\end{aligned}
$$

and

$$
\begin{aligned}
& f_{n}^{(\mu)}(x+y) \\
& =\sum_{k=0}^{N n}(-1)^{k} \sum_{j=[k / N]}^{n} \frac{j !}{(N j) !}\left(\begin{array}{c}
n \\
j
\end{array}\right)\left(\begin{array}{c}
N j \\
k
\end{array}\right)\left(\frac{\prod_{l=1}^{q}\left(\beta_{l}\right)_{j} \prod_{l=1}^{r}\left(\gamma_{l}\right)_{N j}}{\prod_{l=1}^{p}\left(\alpha_{l}\right)_{j} \prod_{l=1}^{s}\left(\delta_{l}\right)_{N j}}\right) f_{n-j}^{(\mu)}(y) \\
& \quad \cdot{ }_{p+N(s+1)} F_{q+N r}\left[\begin{array}{c}
\Delta(N ;-k),\left(\alpha_{p}\right), \Delta\left[N ;\left(\delta_{s}\right)\right] ; \\
\left(\beta_{q}\right), \Delta\left[N ;\left(\gamma_{r}\right)\right] ;
\end{array}\right]
\end{aligned}
$$

Remark 3. Corollary 3 below, which involves such classical orthogonal polynomials as the Jacobi polynomials $P_{n}^{(\alpha, \beta)}(x)$, the Laguerre polynomials $L_{n}^{(\alpha)}(x)$ and the Hermite polynomials $H_{n}(x)$, can be deduced by suitably specializing Theorem 2 or (alternatively) by directly applying (32) in conjunction with the following known polynomial expansions [12, p. 262, Equation 136 (2); p. 207, Equation 118 (2); p. 194, Equation 110 (5)]:

$$
\begin{gathered}
x^{n}=n ! \sum_{k=0}^{n}(-1)^{k}\left(\begin{array}{c}
n+\alpha \\
n-k
\end{array}\right) \frac{\alpha+\beta+2 k+1}{(\alpha+\beta+k+1)_{n+1}} P_{k}^{(\alpha, \beta)}(1-2 x), \\
x^{n}=n ! \sum_{k=0}^{n}(-1)^{k}\left(\begin{array}{c}
n+\alpha \\
n-k
\end{array}\right) L_{k}^{(\alpha)}(x)
\end{gathered}
$$

and

$$
(2 x)^{n}=\sum_{k=0}^{[n / 2]}\left(\begin{array}{c}
n \\
2 k
\end{array}\right) \frac{(2 k) !}{k !} H_{n-2 k}(x) .
$$

However, in the case of the derivation of the assertion (49) of Corollary 3 below, the use of the known polynomial expansion (46) is seen to provide a much more convenient and straightforward alternative.

Corollary 3. Let $\left\{f_{n}^{(\mu)}(x)\right\}_{n \in \mathbb{N}_{0}}$ be a one-parameter sequence of Appell polynomials generated by (26) with the parameter $\alpha$ replaced by $\mu$. Then each of the 
following addition formulas holds true:

$$
\begin{aligned}
f_{n}^{(\mu)}(x+y)= & \sum_{k=0}^{n}(-1)^{k} \sum_{j=k}^{n} j !\left(\begin{array}{c}
n \\
j
\end{array}\right)\left(\begin{array}{c}
j+\alpha \\
j-k
\end{array}\right) \frac{\alpha+\beta+2 k+1}{(\alpha+\beta+k+1)_{j+1}} \\
& \cdot f_{n-j}^{(\mu)}(y) P_{k}^{(\alpha, \beta)}(1-2 x), \\
f_{n}^{(\mu)}(x+y)= & \sum_{k=0}^{n}(-1)^{k} \sum_{j=k}^{n} j !\left(\begin{array}{c}
n \\
j
\end{array}\right)\left(\begin{array}{c}
j+\alpha \\
j-k
\end{array}\right) f_{n-j}^{(\mu)}(y) L_{k}^{(\alpha)}(x)
\end{aligned}
$$

and

$$
f_{n}^{(\mu)}(x+y)=\sum_{k=0}^{[n / 2]} \sum_{j=2 k}^{n} 2^{-j}\left(\begin{array}{c}
n \\
j
\end{array}\right)\left(\begin{array}{c}
j \\
2 k
\end{array}\right) \frac{(2 k) !}{k !} f_{n-j}^{(\mu)}(y) H_{j-2 k}(x) .
$$

\section{An umbral-calculus generalization of the addition theorems}

Following the notations and conventions described in [13], let $\mathfrak{P}$ be the algebra of polynomials in the single variable $x$ over the field $\mathbb{C}$ of complex numbers. Then the following formal power series:

$$
f(t)=\sum_{k=0}^{\infty} a_{k} \frac{t^{k}}{k !}
$$

defines a linear functional on $\mathfrak{P}$ by setting

$$
<f(t) \mid x^{n}>=a_{n} \quad\left(n \in \mathbb{N}_{0}\right) .
$$

Let $\left\{f_{n}(x)\right\}_{n \in \mathbb{N}_{0}}$ be the Appell sequence corresponding to the function $f(t)$ as in the generating function (10).

Theorem 3. Let $\left\{f_{n}(x)\right\}_{n \in \mathbb{N}_{0}}$ and $\left\{g_{n}(x)\right\}_{n \in \mathbb{N}_{0}}$ be the Appell sequences corresponding to the functions $f(t)$ and $g(t)$, respectively. Then

$$
f_{n}(x+y)=\sum_{k=0}^{n}\left(\begin{array}{l}
n \\
k
\end{array}\right) f_{k}(y) \sum_{j=0}^{n-k} \frac{<g(t) \mid\left(x^{n-k}\right)^{(j)}>}{j !} g_{j}(x),
$$

where $\left(x^{n-k}\right)^{(j)}$ denotes the derivative of $x^{n-k}$ of order $j$.

The Proof of Theorem 3 is a simple consequence of the following two known results (see [13, Theorems 2.5.2 and 2.5.8]).

Lemma 2. Let $h_{n}(x)$ be an Appell sequence for the function $h(t)$. Then, for any polynomial $\mathfrak{p}(x)$,

$$
\mathfrak{p}(x)=\sum_{k \geq 0} \frac{<h(t) \mid \mathfrak{p}^{(k)}(x)>}{k !} h_{k}(x),
$$

where $\mathfrak{p}^{(k)}(x)$ denotes the derivative of $\mathfrak{p}(x)$ of order $k$. 
Lemma 3. The sequence $\left\{h_{n}(x)\right\}_{n \in \mathbb{N}_{0}}$ is an Appell sequence if and only if

$$
h_{n}(x+y)=\sum_{k=0}^{n}\left(\begin{array}{l}
n \\
k
\end{array}\right) h_{k}(y) x^{n-k}=\sum_{k=0}^{n}\left(\begin{array}{l}
n \\
k
\end{array}\right) h_{n-k}(y) x^{k} .
$$

Although it is usually not easy to calculate the coefficients

$$
<g(t) \mid\left(x^{n-k}\right)^{(j)}>
$$

occurring in (52), there is an example for the Hermite polynomials $H_{n}(x)$ which is given in [13, p. 90].

\section{Acknowledgements}

The present investigation was supported, in part, by the Hungarian Academy of Sciences under OTKA Grants T67580 and K75566, the János Bolyai Fellowship and the Project TÁMOP 4.2.1./B- 09/1/KONV-2010-0007 implemented through the New Hungary Development Plan co-financed by the European Social Fund and the European Regional Development Fund.

\section{References}

[1] Abramowitz, M., Stegun, I.A. (eds.): Handbook of Mathematical Functions with Formulas, Graphs, and Mathematical Tables. Applied Mathematics Series, vol. 55. National Bureau of Standards, Washington, D.C. (1964); Reprinted by Dover Publications, New York (1965) (see also [11])

[2] Anshelevich, M.: Appell polynomials and their relatives. Internat. Math. Res. Notices 65, 3469-3531 (2004)

[3] Appell, P.: Sur une classes de polynômes. Ann. Sci. École Norm. (Sér. 2) 9, 119-144 (1880)

[4] Chen, K.-Y., Chyan, C.-J., Srivastava, H.M.: Certain classes of polynomial expansions and multiplication formulas. Math. Comput. Model. 37, 135-154 (2003)

[5] Erdélyi, A., Magnus, W., Oberhettinger, F., Tricomi, F.G.: Higher Transcendental Functions, vols. I and III. McGraw-Hill Book Company, New York (1953, 1955)

[6] Garg, M., Jain, K., Srivastava, H.M.: Some relationships between the generalized Apostol-Bernoulli polynomials and Hurwitz-Lerch zeta functions. Integral Transforms Spec. Funct. 17, 803-815 (2006)

[7] González, B., Matera, J., Srivastava, H.M.: Some $q$-generating functions and associated generalized hypergeometric polynomials. Math. Comput. Model. 34(1-2), 133-175 (2001)

[8] Lin, S.-D., Srivastava, H.M., Wang, P.-Y.: Some expansion formulas for a class of generalized Hurwitz-Lerch zeta functions. Integral Transforms Spec. Funct. 17, 817-827 (2006)

[9] Luo, Q.-M., Srivastava, H.M.: Some generalizations of the Apostol-Genocchi polynomials and the Stirling numbers of the second kind. Appl. Math. Comput. 217, 57025728 (2011)

[10] Luke, Y.L.: The Special Functions and Their Approximations, Vol. I, Mathematics in Science and Engineering, Vol. 53-I. A Series of Monographs and Textbooks. Academic Press, New York (1969) 
[11] Olver, F.W.J., Lozier, D.W., Boisvert, R.F., Clark, C.W. (eds.): NIST Handbook of Mathematical Functions [With 1 CD-ROM (Windows, Macintosh and UNIX)]. U.S. Department of Commerce, National Institute of Standards and Technology, Washington, D. C., 2010. Cambridge University Press, Cambridge (2010) (see also [1])

[12] Rainville, E.D.: Special Functions. Macmillan Company, New York (1960); Reprinted by Chelsea publishing Company, Bronx (1971)

[13] Roman, S.: The Umbral Calculus. Academic Press, New York (1984)

[14] Srivastava, H.M.: A contour integral involving Fox's $H$-function. Indian J. Math. 14, 1-6 (1972)

[15] Srivastava, H.M., Choi, J.: Series Associated with the Zeta and Related Functions. Kluwer Acedemic Publishers, Dordrecht (2001)

[16] Srivastava, H.M., Choi, J.: Zeta and $q$-Zeta Functions and Associated Series and Integrals. Elsevier Science Publishers, Amsterdam (2012)

[17] Srivastava, H.M., Manocha, H.L.: A Treatise on Generating Functions. Halsted Press (Ellis Horwood Limited, Chichester), John Wiley and Sons, New York (1984)

[18] Srivastava, H.M., Pintér, Á.: Remarks on some relationships between the Bernoulli and Euler polynomials. Appl. Math. Lett. 17, 375-380 (2004)

Á. Pintér

Institute of Mathematics

MTA-DE Research Group "Equations Functions and Curves"

Hungarian Academy of Sciences and University of Debrecen

P. O. Box 12, 4010 Debrecen, Hungary

e-mail: apinter@science.unideb.hu

H. M. Srivastava

Department of Mathematics and Statistics

University of Victoria

Victoria, BC V8W 3R4

Canada

e-mail: harimsri@math.uvic.ca

Received: April 17, 2012

Revised: June 4, 2012 